\title{
Visualisation of Cardiac Wall Motion Using MR Images
}

\author{
CL Poh ${ }^{1}$, RI Kitney ${ }^{1}$, RBK Shrestha ${ }^{2}$ \\ ${ }^{1}$ Imperial College London, UK \\ ${ }^{2}$ University of Southern California, USA
}

\begin{abstract}
Myocardial motion is a good indicator of coronary artery disease. In diagnosis relating to arterial disease and post infarct assessment it is important to view and study abnormal wall motion. In this paper we describe a web-based visualization interface to view myocardial motion. We focus on using $4 D$ cardiac MR images to analyze left ventricle wall motion. Using the interface it is possible to compare wall motion at different locations in the left ventricle to study abnormal behaviour. We consider our method to have a number of advantages. (i) The method uses MR datasets - which, by definition, are in 3-D and are acquired using non-ionizing radiation. (ii) The method works with any MR data set (i.e. from any scanner). (iii) Our user interface is fully web-based and, hence, the images can be viewed at any location in the hospital.
\end{abstract}

\section{Introduction}

Myocardial motion is a good indicator of coronary artery disease. Abnormal motion of the heart wall during contraction is often caused by a reduction (or occlusion) in blood flow to a region of myocardium or as a result of dead tissue, following an infarct. This phenomenon often occurs in localised areas of the heart. As a result, in both diagnosis relating to coronary artery disease and post infarct assessment, it is important to view and study abnormal wall motion. Cardiac Magnetic Resonance (MR) imaging is a non-invasive tool that produces 2-D images which can be stacked to form a 3-D volume, with high soft tissue contrast. Furthermore, the images can be acquired over time. This makes 4-D (3-D + time) analysis possible. Hence, cardiac MR imaging is effective in allowing clinicians to study myocardial motion.

Segmentation of the raw MR images, to identify tissue structures of interest, is generally necessary to study the pathology. A number of segmentation methods, designed to extract the myocardium of the left ventricle, have been reported [1-3]. Our segmentation process is based on a semi-automatic approach; this uses a radii search and edge detection method that we have developed.
In this paper we describe a web-based visualisation interface to view myocardial motion. The use of webbased information and communication technology (ICT) is becoming increasingly important in medicine specifically the use of web-based technologies to allow the clinician to gain universal access to a patient's data in real time, anywhere in the hospital, using PCs, MACs etc $[4,5]$. Here we focus on using cardiac MR images to analyse left ventricular wall motion.

\section{Methods}

The design of our web-based visualization interface schema to view cardiac wall motion is illustrated in Figure 1.

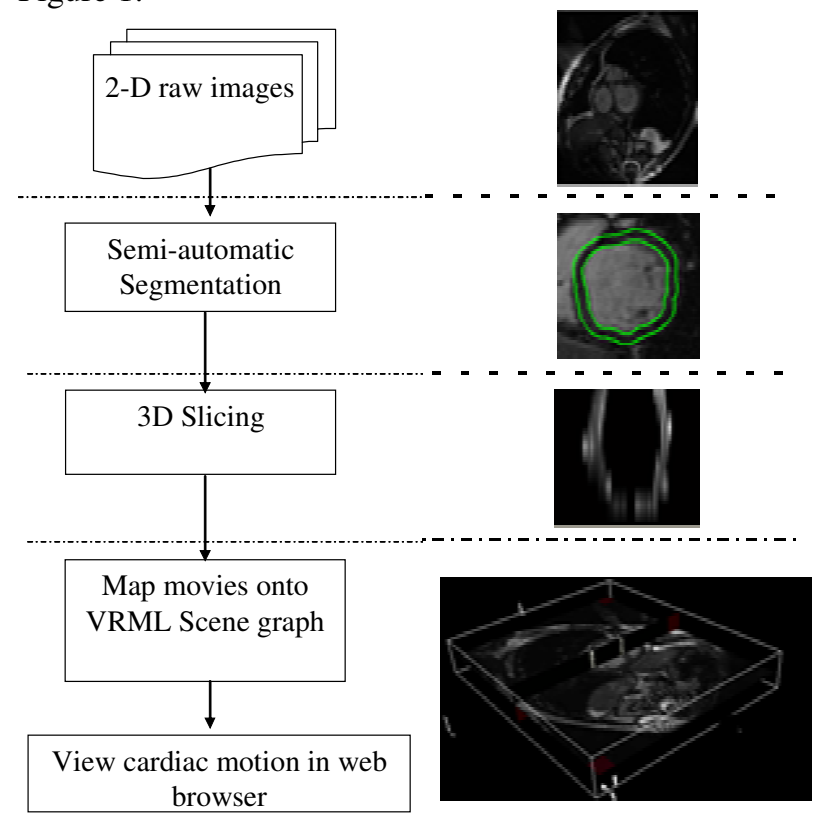

Figure 1. Schema of the web-based visualization interface design to view cardiac wall motion.

Referring to the Figure 1, in the first stage of analysis the cardiac MR volume dataset is segmented to identify the inner wall of the left ventricle. Subsequently, the segmented images are stacked into a 3D volume and resliced in different planes. The resulting images are then converted into moving sequences. These are then mapped 
onto Virtual Reality Markup Language (VRML) scene graph that can be viewed using a web browser.

\subsection{Semi-automatic segmentation}

Our segmentation method was implemented using the Matlab software package. The stages of the segmentation procedure are illustrated in Figure 2.

Referring to the figure, the segmentation process uses a series of radii from an origin defined by the user at the start of the process (see Figure 2a). First, the raw image is filtered using a median filter to remove noise. A radii search from the origin (defined by the user) is then used to find the initial region that encloses the left ventricle (see Figure 2b). As a result, the raw image is masked with the initial region identified. The search procedure then continues in the reverse direction (i.e. inward towards the origin) (see Figure 2c) to detect the inner wall based on a threshold method (i.e. if the pixel intensity for a point is greater than a threshold value, the co-ordinates of the inner wall are stored automatically). This procedure is repeated for $6^{\circ}$ angular increments (i.e. the horizontal) from $0^{\circ}$ to $360^{\circ}$ - to cover the left ventricle in one image. A series of points along the inner wall is obtained after the search method. These points are subsequently joined and smoothed, using a 5 points moving average algorithm, to form a continuous smooth contour along the boundary. An outer contour, a fixed distance (i.e. 5 pixels width) from the inner contour, is created to form the outer wall. This segmentation procedure is performed automatically for all images in the 4D cardiac MR volume dataset.

\subsection{3-D Re-slicing and visualization}

The segmented 2-D images are stacked into a 3-D volume (as shown in Figure 3). Referring to Figure 3, the stack is then re-sliced in different planes (e.g. orthogonal planes - X-X', Y-Y' and non-orthogonal planes - A-A') depending on the requirements of the clinician. This procedure was implemented using the Visualization ToolKit (VTK) [6]. The images generated by the reslicing procedure are then converted into movies and mapped into a Virtual Reality Markup Language (VRML) scene graph.

A VRML scene graph was written to allow the movies to be displayed in a 3-D space and manipulated for the purposes of viewing. One rotating non-orthogonal plane was created using the VRML dragging sensor (i.e. Sphere Sensor). This allows the plane to be dragged with a pointing device (here, a mouse). The movies are then mapped to the plane using the VRML texture mapping feature and inline function. Based on the angle of rotation of the plane, the movies are updated. As a result, the movies that show the cardiac motion at different locations in the left ventricle can be viewed in a 3-D space and displayed in web-browser with a VRML plug-in.

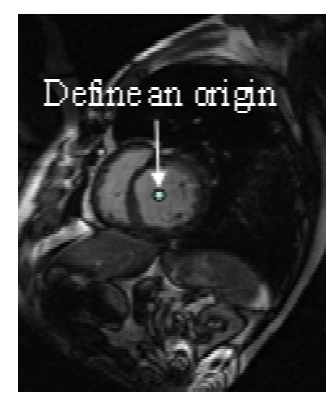

(a)

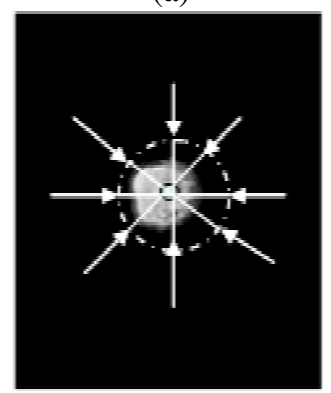

(c)

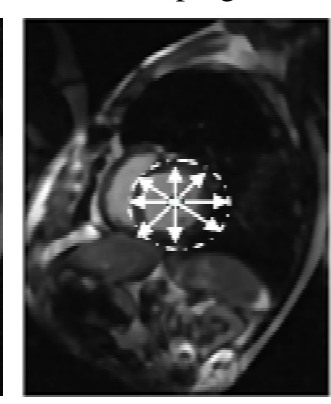

(b)

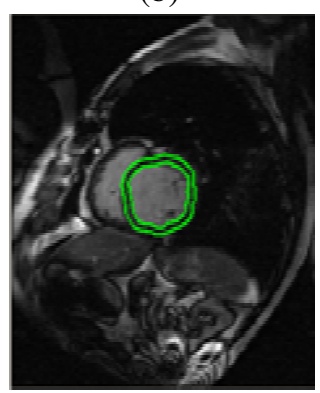

(d)
Figure 2. Stages in the segmentation of a cardiac MR image. Top row: (a) Raw greyscale image, 208 x 256 pixel. (b) Image produced by median filtering of the raw image. Bottom row: (c) Image produced after masking the filtered image with the initial region identified. The search then continues inward towards the origin to identify the inner wall. (d) Final contours of the inner wall of the left ventricle detected.

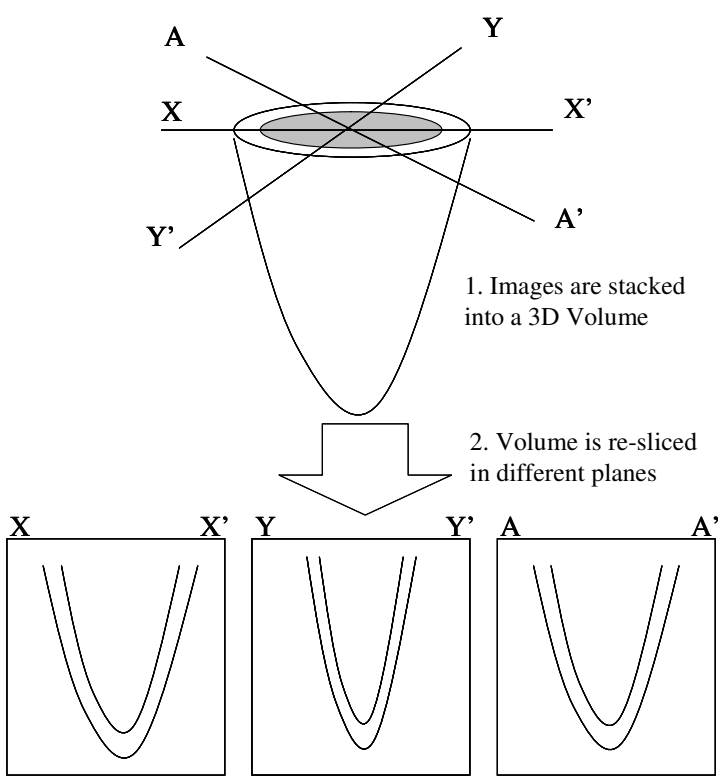

Figure 3. Illustration of 3D Slicing at different locations of the left ventricle. 


\section{Results}

One MR cardiac dataset was segmented and visualized. The dataset comprised 4 slices and 16 frames for each slice (i.e. total of 64 images). Each image had a resolution of $208 \times 256$ pixels. The results of the segmentation for one slice of the dataset are shown in Figure 4.
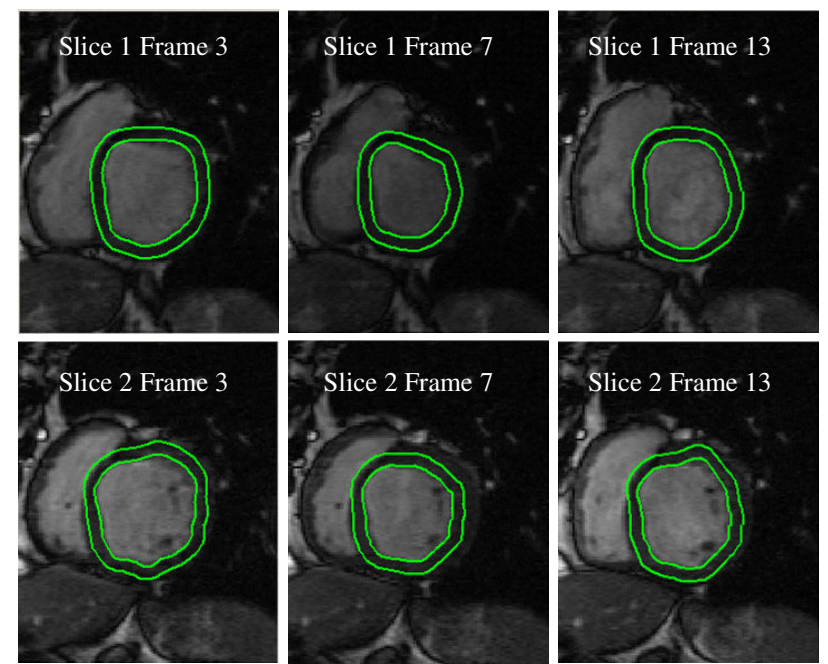

Figure 4. Examples of segmentation results for two slices of the dataset (only frame 3/7/13 are shown).

The wall motion of the left ventricle was visualized using a web-browser with a VRML plug-in. This allows the entire sequence to be viewed as a movie. Figure 5 shows an example of a rendered scene using the viewing interface. With the interface it is possible to slide the plane within the $3-\mathrm{D}$ volume (using a mouse) while the movie is playing. Hence, wall motion at different locations in the left ventricle can be compared to study abnormal behaviour.

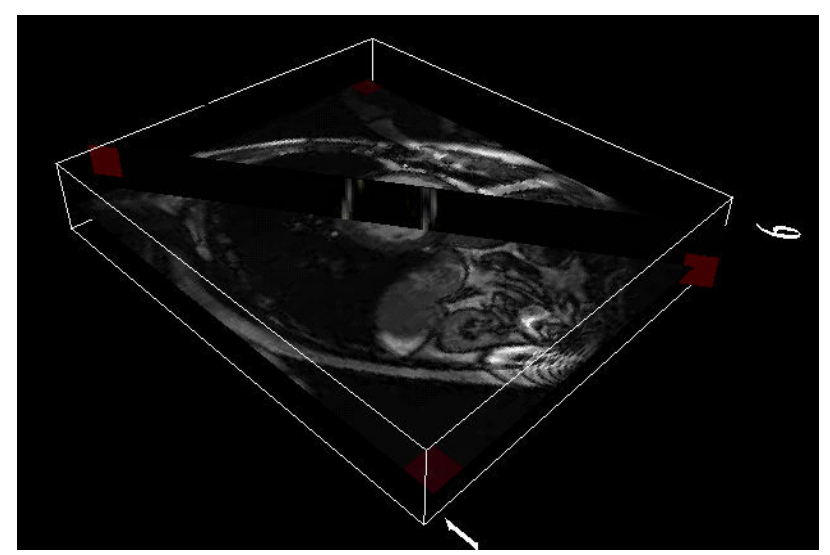

Figure 5. Visualization of cardiac MR dataset in 3D space.

\section{Discussion and conclusions}

A web-based visualization interface for viewing cardiac wall motion was presented. We consider our method to have a number of advantages. (i) The method uses MR datasets - which, by definition, are in 3-D and are acquired using non-ionizing radiation. (ii) The method works with any MR data set (i.e. from any scanner). (iii) Our user interface is fully web-based and, hence, the images can be viewed at any location in the hospital.

\section{Acknowledgements}

The authors would like to thank Mr Tim Hookway and $\mathrm{Mr}$ Alessandro Monteverde for developing the semiautomatic segmentation process. The authors are grateful for the financial support of the EU Similar Network of Excellence in the execution of this project.

\section{References}

[1] Jolly M-P, Duta N, Funka-Lea G. Segmentation of the left ventricle in cardiac MR images. Computer Vision 2001;1:501-508.

[2] Spreeuwers LJ, Breeuwer M. Myocardial boundary extraction using coupled active contours. Computers in Cardiology 2003;30:745-748.

[3] Pluempitiwiriyawej C, Moura JMF, Wu Y-JL, Ho C. STACS: new active contour scheme for cardiac MR image segmentation. IEEE Transactions on Medical Imaging 2005;24:593-603.

[4] Kitney RI, Claesen S, Halls J. A comprehensive web-based patient information environment. Engineering in Medicine and Biology Society 2001;4:3584-5.

[5] Kitney RI, Claesen S, Shrestha RB. An overview of webbased clinical information systems. World Congress on Medical Physics and Biomedical Engineering 2003;4.

[6] Schroeder W, Martin K, Lorensen W. The visualization Toolkit: An Object-Oriented Approach to 3-D Graphics. 2nd ed. NJ: Prentice Hall, 1998.

Address for correspondence

Professor Richard I Kitney

Department of Bioengineering

Imperial College London

Exhibition Road

London SW7 2BX

r.kitney@imperial.ac.uk 